\title{
Increased cardiac distribution of mono-PEGylated Radix Ophiopogonis polysaccharide in both myocardial infarction and ischemia/ reperfusion rats
}

\author{
This article was published in the following Dove Press journal: \\ International Journal of Nanomedicine \\ 9 January 2015 \\ Number of times this article has been viewed
}

\author{
ChunXia Yao ${ }^{1,2, *}$ \\ XiaoLi Shi',* \\ Xiao $\operatorname{Lin}^{1,2}$ \\ Lan Shen' \\ DeSheng $X_{u^{3}}$ \\ Yi Feng ${ }^{2}$
}

'College of Chinese Materia Medica, Shanghai University of Traditional Chinese Medicine, Shanghai, People's Republic of China; ${ }^{2}$ Engineering Research Center of Modern Preparation Technology of TCM of Ministry of Education, Shanghai University of Traditional Chinese Medicine, Shanghai, People's Republic of China; ${ }^{3}$ Shuguang Hospital, Shanghai University of Traditional Chinese Medicine, Shanghai, People's Republic of China

*These authors contributed equally to this work as co-first authors
Correspondence: Xiao Lin College of Chinese Materia Medica, Shanghai University of Traditional Chinese Medicine, I 200 Cailun Road, Pudong, Shanghai 201203, People's Republic of China

Tel/fax +86 2l 5l32 249l

Email duotang@।63.com

Yi Feng

Engineering Research Center of Modern Preparation Technology of TCM of Ministry of Education, Shanghai University of TCM, 1200 Cailun Road, Pudong, Shanghai 201203, People's Republic of China

Tel/fax +86 21 50796201

Emailyy090503@163.com
Abstract: Although PEGylation plays an important role in drug delivery, knowledge about the distribution behavior of PEGylated drugs in ischemic myocardia is rather limited compared to nanoparticles. This work therefore aims to characterize the targeting behavior of the antimyocardial ischemic mono-PEGylated conjugates of Radix Ophiopogonis polysaccharide (ROP) in two clinically relevant animal models, ie, the myocardial infarction (MI) model and the ischemia/reperfusion (IR) model. To determine the effect of the molecular size of conjugates, two representative conjugates (20- and 40-kDa polyethylene glycol mono-modified ROPs), with hydrodynamic size being approximately and somewhat beyond $10 \mathrm{~nm}$, respectively, were studied in parallel at three time points postdose after a method for determining them quantitatively in biosamples was established. The results showed that the cardiac distribution of the two conjugates was significantly enhanced in both MI and IR rats due to the enhanced permeability and retention effect induced by ischemia. In general, the cardiac targeting efficacy of the conjugates in MI and IR rats was approximately 2; however, different changing in targeting efficacy with time was observed between MI and IR rats and also between the conjugates. Although the enhanced permeability and retention effect-based targeting efficacy for mono-PEGylated ROPs was not high, they, as dissolved macromolecules, are prone to diffusion in the cardiac interstitium space, and thus, facilitate the drug to reach perfusion-deficient and nonperfused areas. These findings are helpful in choosing the cardiac targeting strategy.

Keywords: heart distribution, myocardial ischemia, polyethylene glycol, Radix Ophiopogonis polysaccharide

\section{Introduction}

Ischemic heart disease due to coronary atherosclerosis or spasm is one of the leading causes of death and disability worldwide. Coronary heart disease alone caused approximately one of every six deaths in the United States in $2010 .{ }^{1}$ There are a variety of anti-myocardial ischemic drugs and adjuvants; however, many exhibit a short blood half-life and/or poor water solubility, which limits their accumulation in the ischemic regions of the heart. ${ }^{2-4}$ Furthermore, some compounds, such as adenosine and vascular endothelial growth factor, have been shown to induce severe systemic side effects., ${ }^{2,5}$ To maximize their efficacy while minimize adverse effects, such drugs should be efficiently delivered to and retained in the ischemic regions.

Myocardial ischemia, on the one hand, causes a significant decrease in blood flow (the ratio of blood flow in the normal to ischemic myocardial region was reported to be approximately $10: 1^{6}$ ), making efficient local drug delivery challenging. On the 
other hand, it causes some targeting-facilitated pathophysiological changes, especially the enhanced permeability and retention (EPR) effect. ${ }^{7,8}$ Both various nano-sized insoluble drug delivery systems (eg, liposomes, micelles, nanoparticles, microbubbles, and cell ghosts) and soluble polymeric drugs are now used to target parenteral drugs to ischemic myocardia by the EPR effect alone or in combination with certain active and/or physicochemical targeting mechanism/ mechanisms. ${ }^{9-11}$ By comparison, polymeric drugs, which generally have a smaller size than the former, could achieve the longer retention time in the blood and higher drug stability, as well as better drug release and targeting behaviors. ${ }^{12}$ In particular, PEGylation, the process of covalent attachment of one or more polyethylene glycol (PEG) chains to a drug molecule, has been considered as the method of choice for improving the pharmacokinetics and stability of parenteral agents. ${ }^{13,14}$ However, there are yet few reports on using PEGylation as a way for cardiac drug delivery.

Radix Ophiopogonis polysaccharide (ROP), a natural fructan with a molecular weight of $4.8 \mathrm{kDa}$, has unique antimyocardial ischemic activities. ${ }^{15,16}$ It was found ${ }^{17}$ that the accumulation of ROP (hydrodynamic size: $\sim 2 \mathrm{~nm}$ ) in the hearts of rats with experimental myocardial ischemia was approximately 2.2-fold higher than that in normal rat hearts, suggesting that the EPR effect caused by ischemia can surpass the negative effect of the decreased blood flow on drug distribution during ischemia. However, the total amount of ROP distributed in the heart was extremely low due to the rapid renal excretion of ROP (blood half-life: $\sim 0.5$ hours $^{18}$ ). To combat this, ROP was mono-PEGylated successfully with 20-, 30-, or 40-kDa PEG to markedly improve its pharmacokinetic behaviors, without sacrificing therapeutic efficacy. ${ }^{19}$ When administrated at the same molar dose of $4 \mu \mathrm{mol} / \mathrm{kg}$ per injection as ROP, the conjugates could achieve comparable or even better therapeutic effects although their administration intervals were 2- to 6-fold longer than those of ROP. ${ }^{19}$ In addition, it was found in a mouse model of myocardial ischemia established by isoprenaline injection that by mono-PEGylating with 20-kDa PEG, the distribution of ROP in ischemic hearts increased by approximately 30 -fold, and was approximately 1.6-fold greater than in normal hearts owing to the EPR effect caused by ischemia. ${ }^{20}$ However, yet unknown is the cardiac distribution of mono-PEGylated ROPs in two clinically more-relevant circumstances, ie, 1) myocardial infarction (MI) caused by the long-term coronary artery blockage and 2) ischemia/reperfusion (IR), which refers to the renewal of blood flow to ischemic myocardia with the use of thrombolytic therapy or primary percutaneous intervention after an ischemic episode.
The goal of this study was therefore to further characterize the passive targeting behaviors of mono-PEGylated ROPs in both MI and IR. Permanent ligation and transient ligation of the left coronary artery were adopted to induce the aforementioned two clinically relevant pathological statuses of myocardial ischemia in rats, respectively. To determine the effect of the molecular size of conjugates, two representative conjugates (ie, 20- and 40-kDa PEG mono-modified ROPs), whose values are around the midpoint and the upper inflection point of the sigmoidal-shaped profile of plasma half-lives versus apparent molecular weights, respectively, ${ }^{21}$ were studied in parallel at three time points postdose after a sensitive and specific method for determining them quantitatively in biosamples was established.

\section{Materials and methods Materials and animals}

ROP was prepared according to a previous report. ${ }^{22}$ Linear amino-terminated PEG methyl ether (mPEG- $\mathrm{NH}_{2}$ ) hydrochlorides, with molecular weights of 20 - and $40-\mathrm{kDa}$, were purchased from Jenkem Technology Co., Ltd. (Beijing, People's Republic of China). $p$-nitrophenyl chloroformate and 4-N,N-(dimethylamino) pyridine were purchased from Fluka (Buchs, Germany). Fluorescein isothiocyanate (FITC) was purchased from Sigma-Aldrich Co. (St Louis, MO, USA). Extra dry dimethyl sulfoxide, dichloromethane $\left(\mathrm{CH}_{2} \mathrm{Cl}_{2}\right)$, and pyridine were purchased from Acros Organics (Geel, Belgium). All the other chemicals were of reagent grade and were purchased from commercial sources.

Male Sprague Dawley rats, weighing 200-250 g, were supplied by the Lab Animal Center of Shanghai University of Traditional Chinese Medicine (Shanghai, People's Republic of China). They were kept in an environmentally controlled breeding room for at least 4 days before starting the experiments and were fed with standard laboratory food and water ad libitum. The Animal Ethical Experimentation Committee of Shanghai University of Traditional Chinese Medicine approved all the procedures of the animal experiments, which were carried out according to the requirements of the National Act on the Use of Experimental Animals (People's Republic of China).

\section{Preparation of FITC-labeled mono-PEGylated ROPs}

The mono-PEGylated ROPs were synthesized through a moderate coupling reaction between hydroxyl-activated ROP and mPEG-NH $\mathrm{N}_{2}$, according to a previous report. ${ }^{19}$ The molar ratios of hydroxyl-activated ROP to $20-$ and $40-\mathrm{kDa}$ 
mPEG-NH${ }_{2} \mathrm{~s}$ in the reaction were set at 4:1 and 6:1, respectively, to get the mono-PEGylated conjugates. Characterization of the conjugates was carried out by high-performance gel permeation chromatography (HPGPC) in conjunction with anthrone-sulfuric acid colorimetry, according to a previous report. ${ }^{19}$ To determine their levels in biosamples, the conjugates were prelabeled with FITC before administration, according to a method previously established. ${ }^{23}$

\section{Preparation of standard and quality control samples}

Stock solutions of FITC-labeled 20-kDa PEG mono-modified ROP ( $\left.{ }^{\mathrm{F}} \mathrm{MP}_{20 \mathrm{k}}-\mathrm{R}\right)$ and $40-\mathrm{kDa}$ PEG monomodified ROP $\left({ }^{\mathrm{F}} \mathrm{MP}_{40 \mathrm{k}}-\mathrm{R}\right)$ in distilled water at concentrations of 1.002 and $4.08 \mathrm{mg} / \mathrm{mL}$, respectively, were prepared. Two series of standard solutions, with concentrations ranging from 15.66 to $501 \mu \mathrm{g} / \mathrm{mL}$ and 63.75 to $2040 \mu \mathrm{g} / \mathrm{mL}$, were then obtained by further dilution of the two stock solutions with distilled water, respectively.

To prepare the standard calibration samples, a $10-\mu \mathrm{L}$ aliquot of each standard solution was placed in a $1.5-\mathrm{mL}$ centrifuge tube, followed by the addition of $100 \mu \mathrm{L}$ of heart tissue homogenate. After being homogenized thoroughly by vortexing, the mixture was treated according to the sample pretreatment procedure described below. The final standard homogenate concentrations of ${ }^{\mathrm{F}} \mathrm{MP}_{20 \mathrm{k}}-\mathrm{R}$ and ${ }^{\mathrm{F}} \mathrm{MP}_{40 \mathrm{k}}-\mathrm{R}$ were $1.42-91.09 \mu \mathrm{g} / \mathrm{mL}$ and $5.80-370.91 \mu \mathrm{g} / \mathrm{mL}$, respectively. The quality control (QC) samples, which were used in method validation, were prepared in the same way as the standard calibration samples.

\section{Sample pretreatment procedure and analytical conditions}

For pretreating heart homogenates, $21.5 \mu \mathrm{L}$ of $1 \mathrm{M}$ perchloric acid was added to a $50-\mu \mathrm{L}$ supernatant obtained by centrifuging a $100-\mu \mathrm{L}$ portion of each homogenate. The mixture was vortexed and then centrifuged at 10,000 rpm for 2 minutes to precipitate denatured proteins. A $50-\mu \mathrm{L}$ portion of the supernatant was transferred to a new tube and neutralized by the addition of $16 \mu \mathrm{L}$ of $1 \mathrm{M} \mathrm{NaOH}$. After another centrifugation, the supernatant was assayed by the method described below. For pretreating plasma samples, $40 \mu \mathrm{L}$ of $1 \mathrm{M}$ perchloric acid was added to a $100-\mu \mathrm{L}$ portion of each plasma sample to precipitate plasma proteins. An $80-\mu \mathrm{L}$ portion of the supernatant obtained was then neutralized by the addition of $30 \mu \mathrm{L}$ of $1 \mathrm{M} \mathrm{NaOH}$. After the second centrifugation, the supernatant was analyzed by the same method.
The assay system consisted of an Agilent 1200 series HPLC (Agilent Technologies, Santa Clara, CA, USA) with a fluorescence detector set at $\lambda_{\mathrm{ex}} 495 \mathrm{~nm}$ and $\lambda_{\mathrm{em}} 515 \mathrm{~nm}$. Samples were separated by HPGPC using a Shodex OHpak SB-803 HQ column. The eluent was $0.1 \mathrm{M}$ phosphate buffer ( $\mathrm{pH} 7.4$ ), delivered at a flow rate of $0.5 \mathrm{~mL} / \mathrm{min}$. The chromatographic procedures were performed at $30^{\circ} \mathrm{C}$. The volume of injection was $10 \mu \mathrm{L}$.

\section{Preparation of rat models of $\mathrm{MI}$ and IR}

The MI and IR were induced by permanent ligation and transient ligation of the left coronary artery, respectively, as described previously. ${ }^{24}$ In brief, after being anesthetized by intraperitoneal injection of $10 \%$ chloral hydrate, rats were intubated and ventilated mechanically. A left intercostal thoracotomy was performed to expose the heart. The rat model of MI was induced by permanent ligation of the left coronary artery with a 4-0 silk suture. To prepare the rat model of IR, coronary perfusion was restored by loosening the suture after transient ligation of the left coronary artery for 30 minutes. The ischemic condition was confirmed by evidence of immediate changes, including sudden pallor, distinct dilatation, and paralysis of the affected portion of the left ventricle.

\section{Measurement of infarct sizes}

The infarcted area was determined by triphenyltetrazolium chloride (TTC) staining in both MI and IR rats. Briefly, the heart was harvested and rinsed with normal saline. The excised left ventricle was frozen at $-20^{\circ} \mathrm{C}$ for 30 minutes, and then sectioned from apex to base into approximately $2-\mathrm{mm}$ slices. The slices were incubated in a solution of $1 \%$ TTC in phosphate buffered saline ( $\mathrm{pH} \mathrm{7.4)}$ ) at $37^{\circ} \mathrm{C}$ for 15 minutes in darkness, and then fixed in $10 \%$ formaldehyde. The slices were photographed the next day by a digital camera. The infarcted (TTC nonstained) area was isolated from the rest of the cardiac tissue, which was stained red by TTC. The infarcted and normal tissues were separately weighed and the infarct size was expressed as a percentage of mass of the left ventricle.

\section{Cardiac distribution}

Rats in normal, MI, and IR groups were given ${ }^{\mathrm{F}} \mathrm{MP}_{20 \mathrm{k}}-\mathrm{R}$ or ${ }^{\mathrm{F}} \mathrm{MP}_{40 \mathrm{k}}-\mathrm{R}$ at the same molar dose of $4 \mu \mathrm{mol} / \mathrm{kg}$ via the tail vein and were sacrificed at 3,12 , and 24 hours postdose (4-5 rats in each group were sacrificed at each time point). The conjugates were injected at the 3 rd day post-MI; however, they were administered immediately after the IR surgery finished. Blood samples were collected into heparin-containing 
tubes from the abdominal aorta and immediately centrifuged at 10,000 rpm for 10 minutes. The separated plasma was stored at $-20^{\circ} \mathrm{C}$ for subsequent analysis. Meanwhile, the hearts were excised, rinsed with saline solution to wash away the residual blood, and blotted on filter paper. The left ventricle wall was isolated from the rest of the cardiac tissue, and then homogenized by a 3 -fold volume $(\mathrm{m} / \mathrm{v})$ of phosphate buffered saline ( $\mathrm{pH}$ 7.4). The heart homogenates were stored at $-20^{\circ} \mathrm{C}$ for subsequent analysis.

\section{Data analysis}

Data are presented as mean \pm standard deviation. One-way analysis of variance was used for comparisons among groups. Least squares deconvolution multiple comparison was performed between means when equal variances were assumed, while Dunnett's T3 was used when equal variances were not assumed. In all analyses, $P<0.05$ was considered to indicate statistical significance.

\section{Results}

\section{Method validation}

The specificity of the method was accessed by the inspection of chromatograms derived from processed blank and cardiac homogenate samples. The results showed that both ${ }^{\mathrm{F}} \mathrm{MP}_{20 \mathrm{k}}-\mathrm{R}$ and ${ }^{\mathrm{F}} \mathrm{MP}_{40 \mathrm{k}}-\mathrm{R}$ were well separated from the other substances, and no interferences were detected (Figure 1). The typical retention times for ${ }^{\mathrm{F}} \mathrm{MP}_{20 \mathrm{k}}-\mathrm{R}$ and ${ }^{\mathrm{F}} \mathrm{MP}_{40 \mathrm{k}}-\mathrm{R}$ were approximately 15.1 and 13.9 minutes, respectively.

Standard curves of the peak height $(\mathrm{Y})$ to the concentration $(\mathrm{C})$ were constructed using the $1 / \mathrm{C}$ weighted linear least squares regression model. The linearity was evident over the concentration ranges studied, with the correlation coefficients larger than 0.999 (Table 1). The detection limit and quantification limit of the method were calculated from the signal-to-noise ratio of 3 and 10, respectively. The results are listed in Table 1, which indicate that the method is sensitive enough for this study.

The accuracy and precision of the method were examined by adding the known amounts of reference standards to blank heart tissue homogenates. The accuracy describes the closeness of test results obtained by the method to the true value of the analyte and the precision is the coefficient of variation of test results. The results showed that the method was accurate and precise, with the relative recoveries ranging from $94 \%$ to $101 \%$ and the within- and inter-batch relative standard deviation values less than $16 \%$ (Table 2). The absolute recoveries of ${ }^{\mathrm{F}} \mathrm{MP}_{20 \mathrm{k}}-\mathrm{R}$ and ${ }^{\mathrm{F}} \mathrm{MP}_{40 \mathrm{k}}-\mathrm{R}$, which were tested at three QC levels by comparing the peak heights of pretreated heart tissue homogenates with those obtained by direct injection of standard solutions at the same concentrations, were between $48 \%$ and $66 \%$ (Table 2).

The stability of ${ }^{\mathrm{F}} \mathrm{MP}_{20 \mathrm{k}}-\mathrm{R}$ and ${ }^{\mathrm{F}} \mathrm{MP}_{40 \mathrm{k}}-\mathrm{R}$ in cardiac tissue homogenates was investigated under a variety of storage and process conditions, including being kept at room temperature for 24 hours, in a refrigerator $\left(-20^{\circ} \mathrm{C}\right)$ for 4 days, and undergoing two freeze-thaw cycles. The ratios of the assay results of QC samples stored under different conditions to those freshly prepared were within $90 \%-115 \%$.

As a whole, the method established was confirmed to be qualified for this study. In addition, the method used for determining plasma samples was also validated in our previous reports. $^{21,25}$

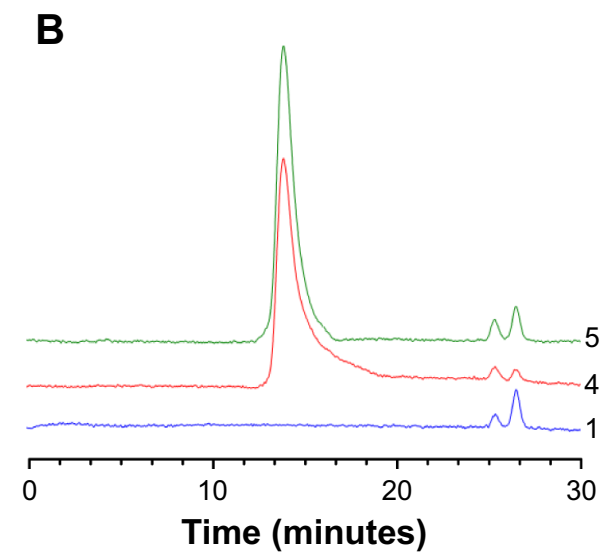

Figure I Representative chromatograms.

Notes: Representative chromatograms for the determination of ${ }^{\mathrm{F} M P}{ }_{20 \mathrm{k}}-\mathrm{R}(\mathbf{A})$ and $\mathrm{FMP}_{40 \mathrm{k}} \mathrm{R}(\mathbf{B})$ in the heart tissue by HPGPC. I= blank heart tissue; $2=$ blank heart tissue spiked with standard $\mathrm{FMP}_{20 \mathrm{k}}-\mathrm{R}$ solution; $3=$ heart tissue sample collected at 3 hours after intravenous administration of $\mathrm{FMP}_{20 \mathrm{k}}-\mathrm{R} ; 4=$ blank heart tissue spiked with standard $\mathrm{FMP}_{40 \mathrm{k}}-\mathrm{R}$ solution; $5=$ heart tissue sample collected at 3 hours after intravenous administration of $\mathrm{FMP}_{40 \mathrm{k}}-\mathrm{R}$.

Abbreviations: ${ }^{\mathrm{F} M P} \mathrm{P}_{20 \mathrm{k}}-\mathrm{R}, 20-\mathrm{kDa}$ PEG mono-modified ROP; $\mathrm{FMP}_{40 \mathrm{k}}-\mathrm{R}, 40-\mathrm{kDa}$ PEG mono-modified ROP; HPGPC, high-performance gel permeation chromatography; PEG, polyethylene glycol; ROP, Radix Ophiopogonis polysaccharide. 
Table I Linearity and sensitivity of detection for $\mathrm{FMP}_{20 \mathrm{k}}-\mathrm{R}$ and $\mathrm{FMP}_{40 \mathrm{k}}-\mathrm{R}$ in rat myocardia by HPGPC ( $\mathrm{n}=7$ )

\begin{tabular}{llllll}
\hline Samples & Standard curves & Linear ranges $(\mu \mathrm{g} / \mathrm{mL})$ & Correlation coefficients & $\mathrm{DL}(\mu \mathrm{g} / \mathrm{mL})$ & $\mathrm{QL}(\mu \mathrm{g} / \mathrm{mL})$ \\
\hline $\mathrm{FMP}_{20 \mathrm{~K}}-\mathrm{R}$ & $\mathrm{H}=54.867 \mathrm{C}-0.05 \mathrm{I}$ & $\mathrm{I} .42-91.09$ & 0.9997 & 0.28 & 0.94 \\
$\mathrm{FMP}_{40 \mathrm{~K}}-\mathrm{R}$ & $\mathrm{H}=33.212 \mathrm{C}-0.083$ & $5.79-370.91$ & 0.9994 & 0.44 & 1.45 \\
\hline
\end{tabular}

Abbreviations: DL, detection limit; ${ }^{\mathrm{F} M P}{ }_{20 \mathrm{k}}-\mathrm{R}$, 20-kDa PEG mono-modified ROP; ${ }^{\mathrm{M} M \mathrm{P}_{40 \mathrm{~K}}} \mathrm{-R}, 40-\mathrm{kDa}$ PEG mono-modified ROP; HPGPC, high-performance gel permeation chromatography; PEG, polyethylene glycol; QL, quantification limit; ROP, Radix Ophiopogonis polysaccharide.

\section{Measurement of infarct sizes}

The infarct size in MI rats suffering from 4-day ligation of the left coronary artery was $17.3 \% \pm 4.2 \%(n=4)$, and the value in IR rats suffering from transient 30-minute ligation and subsequent 24 -hour reperfusion was $34.5 \% \pm 7.7 \%(\mathrm{n}=3)$. The representative photographs of transverse slices following TTC staining are shown in Figure 2.

\section{Cardiac distribution of $\mathrm{FMP}_{20 \mathrm{k}}-\mathrm{R}$ and $\mathrm{FMP}_{40 \mathrm{k}}-\mathrm{R}$}

Heart and plasma levels of the conjugates in normal, MI, and IR rats at 3,12, and 24 hours after a single intravenous dose of $4 \mu \mathrm{mol} / \mathrm{kg}$ are listed in Tables 3 and 4 . The results showed that compared to the normal group, the cardiac levels of the conjugates significantly $(P<0.01)$ increased in both MI and IR groups at each time point postdose. The plasma levels of the two conjugates in the MI group were significantly $(P<0.05$ or $P<0.01)$ lower than the normal group at 12 hours postdose and the plasma level of ${ }^{\mathrm{F}} \mathrm{MP}_{20 \mathrm{k}}-\mathrm{R}$ was also significantly $(P<0.05)$ lower at 3 hours postdose. The tendency of cardiac distribution, expressed as the ratio of cardiac level to plasma level of the conjugate at each time point, was also compared among groups. The distribution tendency of ${ }^{\mathrm{F}} \mathrm{MP}_{20 \mathrm{k}}-\mathrm{R}$ was higher than that of ${ }^{\mathrm{F}} \mathrm{MP}_{40 \mathrm{k}}-\mathrm{R}$ in each group, and both of them were significantly higher in MI and IR rats than in normal rats (Figure 3; Tables 5 and 6). In general, the ischemic heart targeting efficacy of the conjugates in MI and IR rats was around 2 (Tables 5 and 6). Different changing in targeting efficacy with time was observed between MI and IR rats and also between ${ }^{\mathrm{F}} \mathrm{MP}_{20 \mathrm{k}}-\mathrm{R}$ and ${ }^{\mathrm{F}} \mathrm{MP}_{40 \mathrm{k}}-\mathrm{R}$ (Figure 4), which, together with the distribution tendency, is discussed in detail in the following section.

\section{Discussion}

It was well known that macromolecular and nanoparticle carriers were capable of passively accumulating in solid tumors via the EPR effect. ${ }^{26,27}$ Similar to tumors, the EPR effect was also found in ischemic heart diseases in limited researches. Since the EPR effect is size-dependent and the "cut-off" size of the permeabilized vasculature varies from case to case, the size of carriers may control the efficacy of passive delivery to ischemic hearts. ${ }^{28}$ It was reported that liposomes (mean diameter $\sim 134 \mathrm{~nm}$ ) were capable of accumulating in the border (the noninfarcted areas at risk) as well as the infarcted myocardia, but not in the nonischemic ones at 3 hours postdose in a rat model of IR. ${ }^{2}$ Micelles $(7-20 \mathrm{~nm}$ ) have been shown to passively accumulate in the ischemic myocardia at an efficiency of approximately eight times higher than in the adjacent normal zone at 3 hours postdose in a rabbit model of IR. ${ }^{8}$ In the mouse models of both acute and chronic MI, micelles $(\sim 15 \mathrm{~nm})$ permeated the entire infarct area, which renders them very suited for the local delivery of cardioprotective or antiremodeling drugs. In comparison, liposomes $(\sim 100 \mathrm{~nm})$ displayed slower and more restricted extravasation from the vasculature and are therefore an attractive vehicle for the delivery of proangiogenic drugs. ${ }^{29}$ In the present research, the PEG-ROP conjugates, with a hydrodynamic size approximately or beyond $10 \mathrm{~nm},{ }^{21}$ were studied in parallel on the rat models of both MI and IR to clarify their ability to accumulate in the ischemic myocardia via the EPR effect.

Table 2 Recovery, accuracy, and precision for the assay of $\mathrm{FMP}_{20 \mathrm{k}}-\mathrm{R}$ and $\mathrm{FMP}_{40 \mathrm{k}}-\mathrm{R}$ in rat myocardia by HPGPC ( $\mathrm{n}=5$ )

\begin{tabular}{llllll}
\hline Samples & $\begin{array}{l}\text { Added concentration } \\
(\mu \mathrm{g} / \mathrm{mL})\end{array}$ & Recovery (\%) & Accuracy (\%) & \multicolumn{2}{l}{ Precision RSD (\%) } \\
\cline { 5 - 6 } & & & & Within-batch & Inter-batch \\
\hline $\mathrm{FMP}_{20 \mathrm{~K}}-\mathrm{R}$ & 2.85 & $55.46 \pm 2.13$ & $97.39 \pm 6.87$ & $10.6 \mathrm{I}$ & 8.87 \\
& 11.39 & $59.49 \pm 3.68$ & $99.94 \pm 2.42$ & 2.63 & 4.76 \\
& 45.55 & $65.91 \pm 1.79$ & $100.78 \pm 1.23$ & 1.24 & 2.04 \\
$\mathrm{FMP}_{40 \mathrm{~K}}-\mathrm{R}$ & 11.59 & $48.02 \pm 5.70$ & $97.72 \pm 9.03$ & 9.20 & 15.65 \\
& 46.36 & $54.16 \pm 5.78$ & $94.08 \pm 9.46$ & 5.93 & 1.75 \\
& 185.45 & $59.74 \pm 4.24$ & $97.85 \pm 6.86$ & 6.69 & 6.09 \\
\hline
\end{tabular}

Abbreviations: $\mathrm{FMP}_{20 \mathrm{k}} \mathrm{R}$, 20-kDa PEG mono-modified ROP; ${ }^{\mathrm{F} M \mathrm{P}_{40 \mathrm{k}} \mathrm{K}}$, 40-kDa PEG mono-modified ROP; HPGPC, high-performance gel permeation chromatography; PEG, polyethylene glycol; ROP, Radix Ophiopogonis polysaccharide; RSD, relative standard deviation. 
A

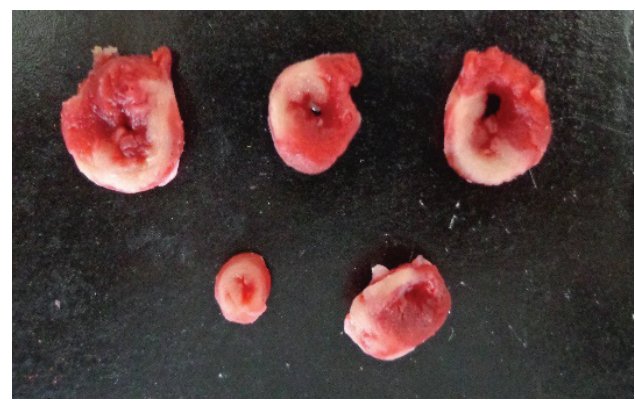

B

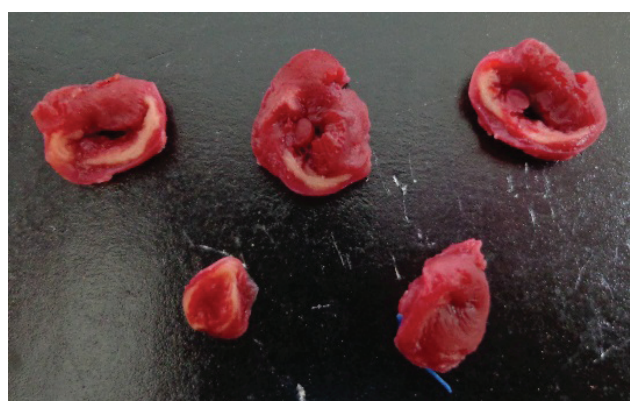

Figure 2 Representative TTC staining photographs.

Notes: Representative TTC staining photographs of heart transverse slices from a rat receiving IR surgery with reperfusion for 24 hours $(\mathbf{A}$, infarct size $=43.4 \%)$ and slices from a rat receiving $\mathrm{Ml}$ surgery with a prolonged period of ischemia for 4 days (B, infarct size $=19.2 \%$ ).

Abbreviations: IR, ischemia/reperfusion; MI, myocardial infarction; TTC, triphenyltetrazolium chloride.

The results showed that the targeting efficacy for $\mathrm{MP}_{20 \mathrm{k}}-\mathrm{R}$ and $\mathrm{MP}_{40 \mathrm{k}}-\mathrm{R}$ was relatively lower, being approximately 2 . This was believed to be due to the linear and flexible structure of the conjugates, which can help them cross the endothelium by a "snake-like" movement. Therefore, with the apparent molecular weight appearing to surpass the pore size of the endothelium, they still can pass through it, although at lower rates. However, unlike insoluble carriers, the PEG-ROP conjugates, as dissolved macromolecules, were prone to diffusion in the cardiac interstitium space, facilitating the drug to reach the perfusion-deficient and nonperfused areas. In addition, it was shown that in the rat MI model, the cardiac distribution tendencies of $\mathrm{MP}_{20 \mathrm{k}}-\mathrm{R}$ and $\mathrm{MP}_{40 \mathrm{k}}-\mathrm{R}$, which were intravenously administrated at day 3 post-MI and allowed to circulate for 24 hours, were 1.95- and 2.57-fold higher than in the normal hearts, respectively. The values were comparable to that found in a previous research where antiP-selectin immunoliposomes, injected 4 hours post-MI and allowed to circulate for 24 hours, showed a $92 \%$ increase in accumulation in the infarcted myocardium compared to the noninfarcted myocardium. ${ }^{30}$ This suggested that PEGylation, as the method of choice for improving the pharmacokinetics and stability of parental agents, ${ }^{13,14}$ might achieve a targeting efficacy comparable to antibody-coated liposomes.

Taking into consideration the following two factors - 1) the increased vascular permeability caused by the IR injury persisted for at least 48 hours and gradually recovered within 2 weeks; ${ }^{31}$ and 2) the therapeutic interventions proven to reduce the infarct size in both experimental and clinical models were often administered at the time of myocardial reperfusion $^{32}$ - the PEG-ROP conjugates were injected via the tail vein immediately after the IR surgery finished and were allowed to circulate for the predetermined amounts of time. For the rat model of MI, rats were subjected to prolonged periods of myocardial ischemia for 3 days without reperfusion. The conjugates were administered at the 3 rd day post-MI because 1) vascular proliferation became quite prominent by day $3,{ }^{33}$ which offered increased possibility of delivering drug to the nonperfused necrotic zone, and 2) the occurrence of death of rats observably decreased at day 3 post-MI, ensuring that the drug-administered rats would survive until being sacrificed.

In general, there are three main factors that would affect drug distribution in the ischemic zone, that is, the tissue perfusion flow rate, the elimination half-life of drug, and the vascular permeability. ${ }^{34}$ The first two determine the drug amount and duration of distribution while the last affects the efficacy of distribution. The distribution pattern of the PEG-ROP conjugates is related to their plasma pharmacokinetics, which may change under the ischemic condition. Therefore, the pharmacokinetics of one of the conjugates (ie, ${ }^{\mathrm{F}} \mathrm{MP}_{20 \mathrm{k}}-\mathrm{R}$ ) in normal, MI, and IR rats was studied previously. The results showed that compared to the normal group, the blood conjugate concentrations in the IR group were significantly higher at the first three time points (ie, 5 minutes, 40 minutes, and 3 hours), but not at the later time points (data not shown), which made the pharmacokinetic parameters including area

Table 3 Concentrations of $\mathrm{FMP}_{20 \mathrm{k}}-\mathrm{R}$ in myocardium and plasma after a single intravenous dose of $4 \mu \mathrm{mol} / \mathrm{kg}$ in rats $(n=4-5)$

\begin{tabular}{|c|c|c|c|c|}
\hline \multirow[t]{2}{*}{ Biosample } & \multirow[t]{2}{*}{ Group } & \multicolumn{3}{|c|}{ Concentration $(\mu \mathrm{mol} / \mathrm{L})$} \\
\hline & & 3 hours & I 2 hours & 24 hours \\
\hline \multirow[t]{3}{*}{ Myocardium } & Normal & $0.535 \pm 0.05 I$ & $0.267 \pm 0.021$ & $0.159 \pm 0.023$ \\
\hline & IR & $1.222 \pm 0.149 * *$ & $0.372 \pm 0.04 I^{* *}$ & $0.227 \pm 0.020^{* *}$ \\
\hline & MI & $0.876 \pm 0.134 * * \ldots$ & $0.384 \pm 0.086 * *$ & $0.332 \pm 0.012^{* * \ldots}$ \\
\hline \multirow[t]{3}{*}{ Plasma } & Normal & $18.11 \pm 1.25$ & $4.54 \pm 0.94$ & $\mathrm{I} .5 \mathrm{I} \pm 0.23$ \\
\hline & IR & $20.42 \pm 5.23$ & $4.22 \pm 0.53$ & $1.63 \pm 0.14$ \\
\hline & MI & $15.14 \pm 0.74 *$ & $3.08 \pm 0.47^{* \# \#}$ & $1.60 \pm 0.07$ \\
\hline
\end{tabular}

Notes: $* * P<0.01$ and $* P<0.05$, compared with the normal group. ${ }^{\#} P<0.01$ and ${ }^{\#} P<0.05$, compared with the IR group.

Abbreviations: ${ }^{F} \mathrm{MP}_{20 \mathrm{k}}-\mathrm{R}, 20-\mathrm{kDa}$ polyethylene glycol mono-modified Radix Ophiopogonis polysaccharide; IR, ischemia/reperfusion; MI, myocardial infarction. 
Table 4 Concentrations of $\mathrm{FMP}_{40 \mathrm{k}}-\mathrm{R}$ in myocardium and plasma after a single intravenous dose of $4 \mu \mathrm{mol} / \mathrm{kg}$ in rats $(n=4-5)$

\begin{tabular}{lllll}
\hline Biosample & Group & \multicolumn{3}{l}{ Concentration $(\mu \mathrm{mol} / \mathrm{L})$} \\
\cline { 3 - 5 } & & $\mathbf{3}$ hours & $\mathbf{I} \mathbf{2}$ hours & $\mathbf{2 4}$ hours \\
\hline Myocardium & Normal & $0.944 \pm 0.105$ & $0.789 \pm 0.078$ & $0.407 \pm 0.064$ \\
& IR & $1.739 \pm 0.233^{* *}$ & $1.159 \pm 0.126^{* *}$ & $0.785 \pm 0.096^{* *}$ \\
& $\mathrm{MI}$ & $1.367 \pm 0.166^{* * * \#}$ & $1.137 \pm 0.188^{* *}$ & $0.894 \pm 0.094^{* *}$ \\
Plasma & Normal & $50.63 \pm 4.53$ & $25.66 \pm 1.70$ & $17.68 \pm 1.33$ \\
& IR & $57.34 \pm 2.14$ & $21.95 \pm 3.89$ & $15.05 \pm 1.70$ \\
& MI & $50.47 \pm 1.62^{\#}$ & $18.78 \pm 2.3 I^{* *}$ & $15.50 \pm 2.61$ \\
\hline
\end{tabular}

Notes: $* * P<0.01$, compared with the normal group. ${ }^{\# P}<0.01$ and $\# P<0.05$, compared with the IR group.

Abbreviations: $\mathrm{FMP}_{40 \mathrm{k}}-\mathrm{R}, 40-\mathrm{kDa}$ polyethylene glycol mono-modified Radix Ophiopogonis polysaccharide; IR, ischemia/reperfusion; MI, myocardial infarction.

under the curve, mean residence time, apparent volume of distribution, and systemic clearance, but not terminal phase half-life, significantly change. These changes should not be completely attributed to the IR injury, because the rats in the IR group were intravenously dosed immediately after IR surgery and before they recovered from anesthesia. Thus, the blood samples at the initial time points were collected from rats that were still in the anesthesia status, which might also lead to pharmacokinetic changes. It was reported that serum lidocaine concentrations were higher in the anesthetized horses than in the awake horses at all time points during lidocaine administration and most pharmacokinetic variables of lidocaine also differed between the two groups. ${ }^{35}$ Therefore, further research is needed to explore the real reason for the observed pharmacokinetic changes here. In the rat MI model, the blood conjugate concentration was significantly less than that in the normal group at 12 hours postdose and also a little lower at the other time points although no significant differences were observed. The apparent volume of distribution in MI rats was a little higher than that in normal rats, which is well related to the lower conjugate plasma concentration at each time point in MI rats. In terms of the fact that plasma drug concentrations would influence its cardiac distribution, the distribution tendency, expressed as the ratio of the cardiac level to the plasma level at the same time point, was compared among normal, MI, and IR rats, which would not only exclude the influence of pharmacokinetic changes on cardiac distribution, and thus truly reflect the targeting efficacy achieved by the EPR effect, but also eliminate the influence of the dose deviation.

In IR, microvascular obstruction has been confirmed to happen in the ischemic regions. The major contributing factors include capillary damage with impaired vasodilatation; external capillary compression by endothelial cell and cardiomyocyte swelling; platelet micro-thrombi; the release of soluble vasomotor and thrombogenic substances; and neutrophil plugging. ${ }^{36}$ This significantly reduces blood circulation in the ischemic regions. Compared to IR, the infarct region is further poorly perfused in acute MI due to no reperfusion. After the acute episode of infarction, a scar forms in an attempt to repair the necrotic area. The infarct area, which is avascular and composed predominately of fibrillar collagen, is enclosed by a border zone which is still somewhat nourished by a vasculature. In chronic MI, although perfusion is partially restored by angiogenesis, ${ }^{37}$ the endothelium of these newly formed blood vessels was shown recently to be highly intact, making its permeability to macromolecules and nano-sized carrier systems rather low. ${ }^{29,38}$ Thus, the distribution of macromolecular drugs to the necrotic core in MI should depend mainly on diffusion from peripheral interstitium, making the total distribution to the ischemic area relatively slow and uneven in MI compared to IR. However, once distributed, macromolecules would retain more efficiently in MI than in IR for the same reason. The above might explain the results
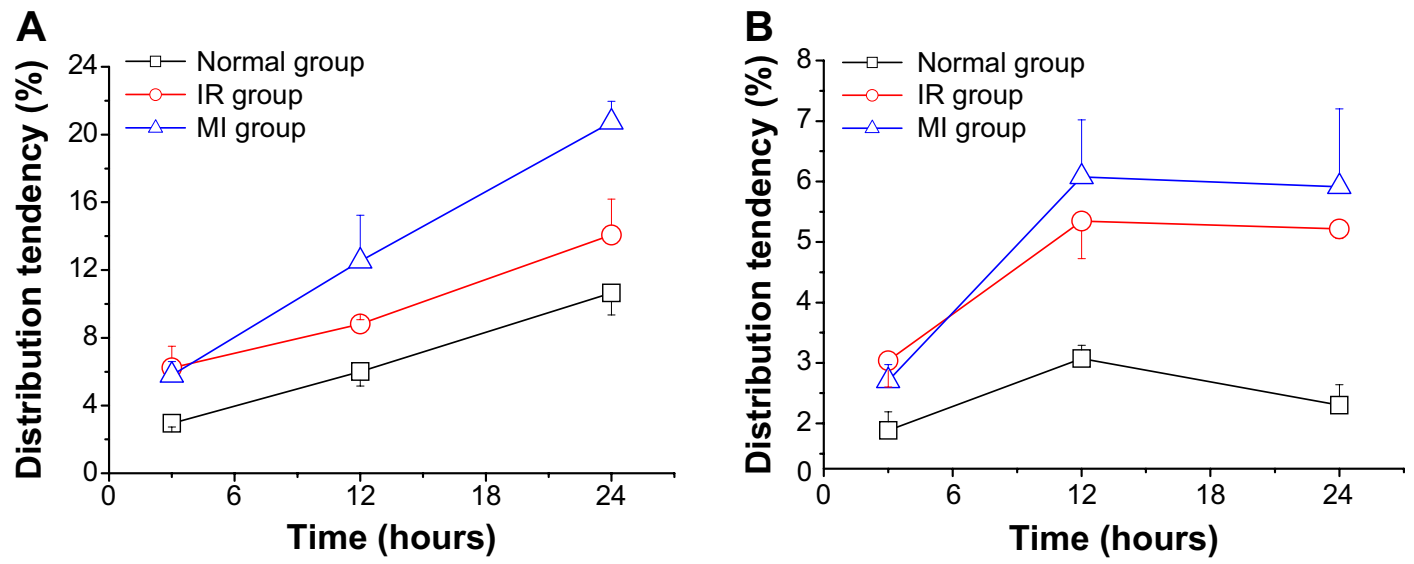

Figure 3 Cardiac distribution tendency.

Note: Cardiac distribution tendency of $\mathrm{FMP}_{20 \mathrm{k}}-\mathrm{R}(\mathbf{A})$ and $\mathrm{FMP}_{40 \mathrm{k}}-\mathrm{R}(\mathbf{B})$ versus time profiles in normal, IR, and $\mathrm{MI}$ rats.

Abbreviations: ${ }^{\mathrm{F} M P_{20 \mathrm{k}}}-\mathrm{R}, 20-\mathrm{kDa}$ PEG mono-modified ROP; ${ }^{\mathrm{F}} \mathrm{P}_{40 \mathrm{k}}-\mathrm{R}, 40-\mathrm{kDa}$ PEG mono-modified ROP; IR, ischemia/reperfusion; MI, myocardial infarction; PEG, polyethylene glycol; ROP, Radix Ophiopogonis polysaccharide. 
Table 5 Cardiac distribution tendency of $\mathrm{FMP}_{20 \mathrm{k}}-\mathrm{R}$ in normal, IR, and $\mathrm{MI}$ rats and cardiac targeting efficacy of $\mathrm{FMP}_{20 \mathrm{k}}-\mathrm{R}$ in $\mathrm{IR}$ and $\mathrm{MI}$ rats at predetermined times postdose $(n=4-5)$

\begin{tabular}{|c|c|c|c|c|c|}
\hline \multirow[t]{2}{*}{ Time } & \multicolumn{3}{|c|}{ Distribution tendency (\%) } & \multicolumn{2}{|c|}{ Targeting efficacy } \\
\hline & Normal & IR & MI & IR & MI \\
\hline $3 \mathrm{~h}$ & $2.96 \pm 0.22$ & $6.22 \pm 1.28 * *$ & $5.79 \pm 0.80 * *$ & $2.10 \pm 0.43$ & $1.96 \pm 0.27$ \\
\hline $12 \mathrm{~h}$ & $6.01 \pm 0.87$ & $8.82 \pm 0.26 * *$ & $12.54 \pm 2.70^{*}$ & $1.47 \pm 0.04^{\$ \$}$ & $2.11 \pm 0.45$ \\
\hline $24 \mathrm{~h}$ & $|0.65 \pm| .3 \mid$ & $14.06 \pm 2.12^{* *}$ & $20.74 \pm 1.22 * * \ldots$ & $1.32 \pm 0.20^{\$ \$}$ & $1.95 \pm 0.11^{88}$ \\
\hline
\end{tabular}

Notes: ${ }^{* * P} P<0.01$ and ${ }^{*} P<0.05$, compared with the normal group; ${ }^{\#} P<0.01$, compared with the IR group; ${ }^{\$} P<0.01$, compared with the targeting efficacy at 3 hours postdose; \&\&P $<0.0$ I, compared with the IR group at 24 hours.

Abbreviations: $\mathrm{FMP}_{20 \mathrm{k}}-\mathrm{R}, 20-\mathrm{kDa}$ polyethylene glycol mono-modified Radix Ophiopogonis polysaccharide; IR, ischemia/reperfusion; MI, myocardial infarction.

observed in this study that at the time point of 3 hours, the targeting efficacy of both $\mathrm{MP}_{20 \mathrm{k}}-\mathrm{R}$ and $\mathrm{MP}_{40 \mathrm{k}}-\mathrm{R}$ in IR was higher than in MI; however, the trend was reversed with the increase of time to 12 and 24 hours.

The change of drug levels in myocardial tissues depends on the compromise of two counteracted courses, ie, drug distribution from blood into the tissue and drug elimination from the tissue. Both of the courses further depend on their respective rate constant $(\mathrm{k})$ and concentration gradient existing between the two sides of transportation if the drug is stable. There are several pathways by which distributed drugs are eliminated from interstitium, including 1) returning back to the blood and 2) transporting into lymphatic vessels by diffusion and tissue cells by pinocytosis.

The reported glomerular filtration thresholds for PEG alone and PEGylated ROP were approximately $30 \mathrm{kDa}^{39,40}$ and $25.4 \mathrm{kDa},{ }^{21}$ respectively. The smaller threshold value for PEGylated ROP was believed to be due to its more spherical shape as well as lower flexibility and deformability in blood than the linear PEG agent alone. As the hydrodynamic sizes of $\mathrm{MP}_{20 \mathrm{k}}-\mathrm{R}$ and $\mathrm{MP}_{40 \mathrm{k}}-\mathrm{R}$ were approximately the same as and somewhat beyond the sieving threshold of the glomerular capillary wall $(\sim 10 \mathrm{~nm}),{ }^{41}$ respectively, $\mathrm{MP}_{20 \mathrm{k}}-\mathrm{R}$ was eliminated from blood approximately 2.5 -fold faster than $\mathrm{MP}_{40 \mathrm{k}}-\mathrm{R}$. Due to faster concentration drop in blood than in myocardial tissues, the cardiac distribution tendency of $\mathrm{MP}_{20 \mathrm{k}}-\mathrm{R}$ increased in both myocardial ischemic and normal rats at all time points studied. While the EPR effect-mediated targeting of long-circulating drugs and drug carriers in tumors took at least several hours to proceed, ${ }^{42,43}$ a maximum or nearly maximum targeting efficacy has been observed for $\mathrm{MP}_{20 \mathrm{k}}-\mathrm{R}$ after only 3 hours postdose both in IR and MI. This should, at least in part, benefit from its relatively small size and, thus, fast diffusion ability. However, the targeting efficacy of $\mathrm{MP}_{20 \mathrm{k}}-\mathrm{R}$ decreased at 12 and 24 hours in IR, indicating that faster-than-normal cardiac tissue elimination happened in IR rather than MI due to its better-than-MI perfused status and higher-than-normal vascular permeability. Compared to $\mathrm{MP}_{20 \mathrm{k}}-\mathrm{R}$, the distribution tendency of $\mathrm{MP}_{40 \mathrm{k}}-\mathrm{R}$ changed differently with time, which increased first and then leveled off. The reason for the initial increase was similar to that for $\mathrm{MP}_{20 \mathrm{k}}-\mathrm{R}$; however, a much smoother cardiac level-time profile was observed for $\mathrm{MP}_{40 \mathrm{k}}-\mathrm{R}$, suggesting a good offset between distribution into and elimination from myocardia. With the blood elimination rate of $\mathrm{MP}_{40 \mathrm{k}}-\mathrm{R}$ decreasing to a certain degree, both blood and heart $\mathrm{MP}_{40 \mathrm{k}}-\mathrm{R}$ levels changed slowly and comparably, resulting in a nearly constant distribution tendency. As for the targeting efficacy, it was shown that more time was needed for larger-in-size $\mathrm{MP}_{40 \mathrm{k}}-\mathrm{R}$ to reach the maximum. In addition, unlike $\mathrm{MP}_{20 \mathrm{k}}-\mathrm{R}$, the targeting efficacy of $\mathrm{MP}_{40 \mathrm{k}}-\mathrm{R}$ did not drop in IR with time. This suggested that the above-mentioned second cardiac elimination pathway, which is blood perfusion-independent, contributed much more significantly to the total cardiac elimination of $\mathrm{MP}_{40 \mathrm{k}}-\mathrm{R}$ due to the reduced vascular penetration ability of $\mathrm{MP}_{40 \mathrm{k}}-\mathrm{R}$. In general, the results obtained here were coincided

Table 6 Cardiac distribution tendency of $\mathrm{FMP}_{40 \mathrm{k}}-\mathrm{R}$ in normal, IR, and $\mathrm{MI}$ rats and cardiac targeting efficacy of $\mathrm{FMP}_{40 \mathrm{k}}-\mathrm{R}$ in IR and $\mathrm{MI}$ rats at predetermined times postdose $(n=4-5)$

\begin{tabular}{|c|c|c|c|c|c|}
\hline \multirow[t]{2}{*}{ Time } & \multicolumn{3}{|c|}{ Distribution tendency (\%) } & \multicolumn{2}{|c|}{ Targeting efficacy } \\
\hline & Normal & IR & MI & IR & MI \\
\hline $3 \mathrm{~h}$ & $|.88 \pm 0.3|$ & $3.04 \pm 0.44 * *$ & $2.7 I \pm 0.27 *$ & $1.61 \pm 0.23$ & $1.44 \pm 0.14$ \\
\hline $12 \mathrm{~h}$ & $3.07 \pm 0.22$ & $5.35 \pm 0.62 * *$ & $6.08 \pm 0.94 *$ & $1.74 \pm 0.20$ & $1.98 \pm 0.31$ \\
\hline $24 \mathrm{~h}$ & $2.30 \pm 0.34$ & $5.22 \pm 0.16 * *$ & $5.91 \pm 1.29 * *$ & $2.26 \pm 0.07^{\$ \$ \#}$ & $2.57 \pm 0.56^{\$ \$}$ \\
\hline
\end{tabular}

Notes: $* * P<0.01$ and $* P<0.05$, compared with the normal group; ${ }^{\$} P<0.01$, compared with the targeting efficacy at 3 hours; $\# p<0.01$, compared with the targeting efficacy at 12 hours.

Abbreviations: $\mathrm{FMP}_{40 \mathrm{k}}-\mathrm{R}, 40-\mathrm{kDa}$ polyethylene glycol mono-modified Radix Ophiopogonis polysaccharide; IR, ischemia/reperfusion; MI, myocardial infarction. 
A

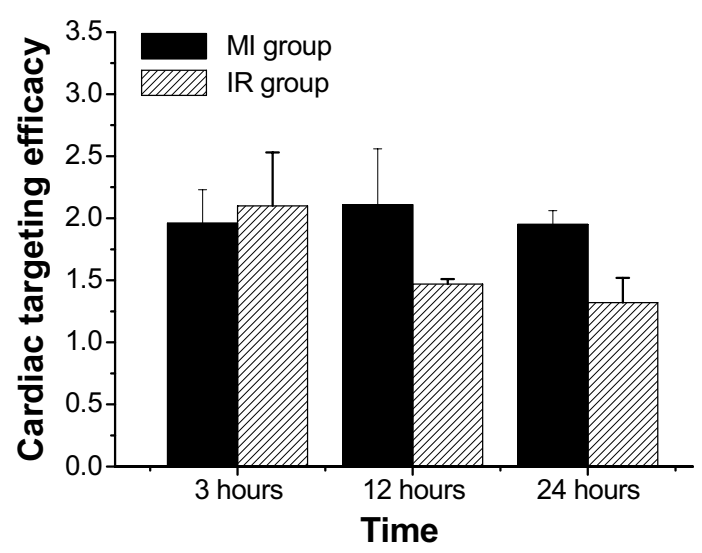

B

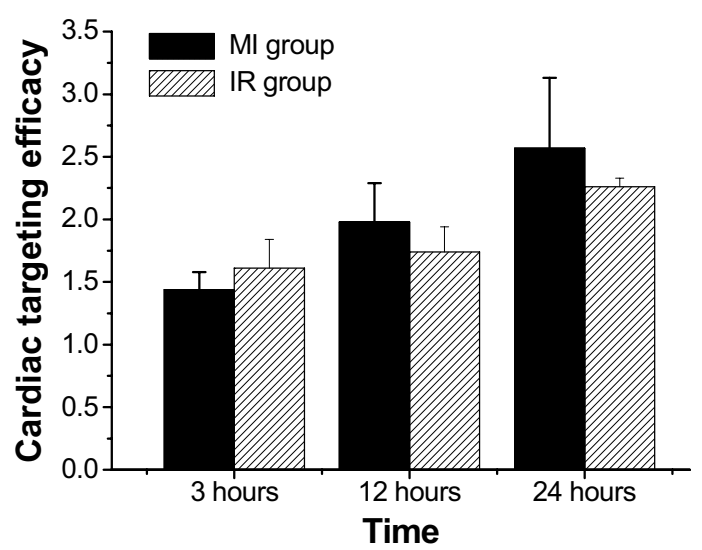

Figure 4 Cardiac targeting efficacy.

Note: Cardiac targeting efficacy of $\mathrm{FMP}_{20 \mathrm{k}} \mathrm{R}(\mathbf{A})$ and $\mathrm{FMP}_{40 \mathrm{k}} \mathrm{R}(\mathbf{B})$ at predetermined times postdose in the rat models of $\mathrm{Ml}$ and IR.

Abbreviations: $\mathrm{FMP}_{20 \mathrm{k}}-\mathrm{R}, 20-\mathrm{kDa}$ PEG mono-modified ROP; $\mathrm{FMP}_{40 \mathrm{k}}-\mathrm{R}, 40-\mathrm{kDa}$ PEG mono-modified ROP; IR, ischemia/reperfusion; MI, myocardial infarction; PEG, polyethylene glycol; ROP, Radix Ophiopogonis polysaccharide.

well with a previous report, ${ }^{34}$ in which the cardiac distribution of 20- and 40-kDa PEGs in normal mice and mice with myocardial ischemia induced by isoprenaline injection was investigated. Therefore, it is reasonable to believe that with quicker distribution to hearts, $\mathrm{MP}_{20 \mathrm{k}}-\mathrm{R}$ is more suitable to treat acute myocardial ischemia, while with a smoother cardiac level-time profile, $\mathrm{MP}_{40 \mathrm{k}}$ - $\mathrm{R}$ is beneficial especially for longterm prophylaxis and/or treatment of myocardial ischemia.

Coronary artery ligation and isoprenaline injection are two commonly used methods to prepare the rat model of myocardial ischemia for the assessment of in vivo activity of drugs. ${ }^{44}$ Though both are effective to induce myocardial ischemia or infarction, circumstances caused by the former are more clinically relevant; namely, it can be used to imitate the clinical status of patients who suffer long-term coronary artery blockage or transient coronary artery blockage followed by reperfusion with the use of thrombolytic therapy or primary percutaneous intervention.

\section{Conclusion}

The cardiac distribution of two mono-PEGylated ROPs $\left(\mathrm{MP}_{20 \mathrm{k}}-\mathrm{R}\right.$ and $\left.\mathrm{MP}_{40 \mathrm{k}}-\mathrm{R}\right)$ was significantly enhanced in both $\mathrm{MI}$ and IR rats due to the EPR effect caused by ischemia. In general, the ischemic heart targeting efficacy of the conjugates in MI and IR rats was approximately 2; however, different changing in targeting efficacy with time was observed between MI and IR rats and also between $\mathrm{MP}_{20 \mathrm{k}}-\mathrm{R}$ and $\mathrm{MP}_{40 \mathrm{k}}-\mathrm{R}$. Although the EPR effect-based targeting efficacy of mono-PEGylated ROPs was not high, they, as dissolved macromolecules with a linear and flexible structure, are prone to diffuse in the cardiac interstitium space, and thus, facilitate the drug to reach the perfusion-deficient and nonperfused areas. These findings are helpful in choosing the targeting strategy for ischemic myocardia.

\section{Acknowledgments}

This work was supported by Program for New Century Excellent Talents in University (NCET-13-0906), the National Natural Science Foundation of China (81073065), the Key Discipline Project of Shanghai Education Committee (J50302), and the "085" Project (085ZY1219) of Shanghai University of TCM.

\section{Disclosure}

The authors report no conflicts of interest in this work.

\section{References}

1. Go AS, Mozaffarian D, Roger VL, et al. Heart Disease and stroke statistics - 2014 update: a report from the American Heart Association. Circulation. 2014;129:e28-e292.

2. Takahama H, Minamino T, Asanuma H, et al. Prolonged targeting of ischemic/reperfused myocardium by liposomal adenosine augments cardioprotection in rats. $J$ Am Coll Cardiol. 2009;53(8):709-717.

3. Verma DD, Hartner WC, Levchenko TS, Bernstein EA, Torchilin VP. ATP-loaded liposomes effectively protect the myocardium in rabbits with an acute experimental myocardial infarction. Pharm Res. 2005; 22(12):2115-2120.

4. Verma DD, Hartner WC, Thakkar V, Levchenko TS, Torchilin VP. Protective effect of coenzyme Q10-loaded liposomes on the myocardium in rabbits with an acute experimental myocardial infarction. Pharm Res. 2007;24(11):2131-2137.

5. Scott RC, Rosano JM, Ivanov Z, et al. Targeting VEGF-encapsulated immunoliposomes to MI heart improves vascularity and cardiac function. FASEB J. 2009;23(10):3361-3367.

6. Mueller TM, Marcus ML, Mayer HE, Williams JK, Hermsmeyer K. Liposome concentration in canine ischemic myocardium and depolarized myocardial cells. Circ Res. 1981;49(2):405-415. 
7. Rodriguez M, Cai WJ, Kostin S, Lucchesi BR, Schaper J. Ischemia depletes dystrophin and inhibits protein synthesis in the canine heart: mechanism of myocardial ischemic injury. J Mol Cell Cardiol. 2005;38(5):723-733.

8. Lukyanov AN, Hartner WC, Torchilin VP. Increased accumulation of PEG-PE micelles in the area of experimental myocardial infarction in rabbits. J Control Release. 2004;94(1):187-193.

9. Sun GL, Lin X. [Mechanisms and strategies for targeting drugs to myocardial ischemic regions]. Үао Хие Хие Вао. 2010;45(7):827-832. Chinese.

10. Torchilin VP. Targeting of drugs and drug carriers within the cardiovascular system. Adv Drug Deliv Rev. 1995;17:75-101.

11. Scott RC, Crabbe D, Krynska B, Ansari R, Kiani MF. Aiming for the heart: targeted delivery of drugs to diseased cardiac tissue. Expert Opin Drug Deliv. 2008;5(4):459-470.

12. Pasut G, Veronese FM. Polymer-drug conjugation, recent achievements and general strategies. Prog Polym Sci. 2007;32(8-9):933-961.

13. Pasut G, Veronese FM. State of the art in PEGylation: the great versatility achieved after forty years of research. $J$ Control Release. 2012;161(2):461-472.

14. Kang JS, DeLuca PP, Lee KC. Emerging PEGyated drugs. Expert Opin Emerg Drugs. 2009;14(2):363-380.

15. Wang S, Zhang Z, Lin X, Xu DS, Feng Y, Ding K. A polysaccharide, MDG-1, induces S1P1 and bFGF expression and augments survival and angiogenesis in the ischemic heart. Glycobiology. 2010;20(4): 473-484.

16. Zheng Q, Feng Y, Xu DS, Lin X, Chen YZ. Influence of sulfation on anti-myocardial ischemic activity of Ophiopogon japonicus polysaccharide. J Asian Nat Prod Res. 2009;11(4):306-321.

17. Lin X, Wang Z, Sun G, Shen L, Xu D, Feng Y. A sensitive and specific HPGPC-FD method for the study of pharmacokinetics and tissue distribution of Radix Ophiopogonis polysaccharide in rats. Biomed Chromatogr. 2010;24(8):820-825.

18. Lin X, Xu DS, Feng Y, Shen L. Determination of Ophiopogon japonicus polysaccharide in plasma by HPLC with modified postcolumn fluorescence derivatization. Anal Biochem. 2005;342(2):179-185.

19. Sun G, Lin X, Shen L, et al. Mono-PEGylated radix ophiopogonis polysaccharide for the treatment of myocardial ischemia. Eur J Pharm Sci. 2013;49(4):629-636.

20. Lin X, Wang ZJ, Wang S, et al. Comparison of tissue distribution of a PEGylated Radix Ophiopogonis polysaccharide in mice with normal and ischemic myocardium. Eur J Pharm Biopharm. 2011;79(3):621-626.

21. Lin X, Wang ZJ, Huang F, et al. Long-circulating delivery of bioactive polysaccharide from radix ophiopogonis by PEGylation. Int $J$ Nanomedicine. 2011;6:2865-2872.

22. Xu DS, Feng Y, Lin X, Deng HL, Fang JN, Dong Q. [Isolation, purification and structural analysis of a polysaccharide MDG-1 from Ophiopogon japonicas]. Yaо Хие Xие Bao. 2005;40(7):636-639. Chinese.

23. Lin X, Wang S, Jiang Y, et al. Poly(ethylene glycol)-Radix Ophiopogonis polysaccharide conjugates: preparation, characterization, pharmacokinetics and in vitro bioactivity. Eur J Pharm Biopharm. 2010;76(2): 230-237.

24. Ye J, Yang L, Sethi R, et al. A new technique of coronary artery ligation: experimental myocardial infarction in rats in vivo with reduced mortality. Mol Cell Biochem. 1997;176(1-2):227-233.

25. Wang ZJ, Lin X, Shen L, Feng Y, Xu DS, Ruan KF. [Pharmacokinetics of a long-circulating PEGylated Radix Ophiopogonis polysaccharide]. Yао Хие Хие Вао. 2011;46(10):1257-1261. Chinese.
26. Torchilin V. Tumor delivery of macromolecular drugs based on the EPR effect. Adv Drug Deliv Rev. 2011;63(3):131-135.

27. Fang J, Nakamura $\mathrm{H}$, Maeda $\mathrm{H}$. The EPR effect: Unique features of tumor blood vessels for drug delivery, factors involved, and limitations and augmentation of the effect. Adv Drug Deliv Rev. 2011;63(3):136-151.

28. Torchilin VP. Drug targeting. Eur J Pharm Sci. 2000;11 Suppl 2: S81-S91.

29. Paulis LE, Geelen T, Kuhlmann MT, et al. Distribution of lipid-based nanoparticles to infarcted myocardium with potential application for MRI-monitored drug delivery. J Control Release. 2012;162(2): 276-285.

30. Scott RC, Wang B, Nallamothu R, et al. Targeted delivery of antibody conjugated liposomal drug carriers to rat myocardial infarction. Biotechnol Bioeng. 2007;96(4):795-802.

31. Horwitz LD, Kaufman D, Keller MW, Kong Y. Time course of coronary endothelial healing after injury due to ischemia and reperfusion. Circulation. 1994;90(5):2439-2447.

32. Oerlemans MI, Koudstaal S, Chamuleau SA, de Kleijn DP, Doevendans PA, Sluijter JP. Targeting cell death in the reperfused heart: pharmacological approaches for cardioprotection. Int J Cardiol. 2013; 165(3):410-422.

33. Fishbein MC, Maclean D, Maroko PR. Experimental myocardial infarction in the rat: qualitative and quantitative changes during pathologic evolution. Am J Pathol. 1978;90(1):57-70.

34. Sun G, Lin X, Hong Y, Feng Y, Ruan K, Xu D. PEGylation for drug delivery to ischemic myocardium: Pharmacokinetics and cardiac distribution of poly(ethylene glycol)s in mice with normal and ischemic myocardium. Eur J Pharm Sci. 2012;46(5):545-552.

35. Feary DJ, Mama KR, Wagner AE, Thomasy S. Influence of general anesthesia on pharmacokinetics of intravenous lidocaine infusion in horses. Am J Vet Res. 2005;66(4):574-580.

36. Hausenloy DJ, Yellon DM. Myocardial ischemia-reperfusion injury: a neglected therapeutic target. J Clin Invest. 2013;123(1):92-100.

37. Ware JA, Simons M. Angiogenesis in ischemic heart disease. Nat Med. 1997;3(2):158-164

38. Dvir T, Bauer M, Schroeder A, et al. Nanoparticles targeting the infarcted heart. Nano Lett. 2011;11(10):4411-4414.

39. Yamaoka T, Tabata Y, Ikada Y. Distribution and tissue uptake of poly(ethylene glycol) with different molecular weights after intravenous administration to mice. J Pharm Sci. 1994;83(4):601-606.

40. Greenwald RB, Gilbert CW, Pendri A, Conover CD, Xia J, Martinez A. Drug delivery systems: water soluble taxol 2'-poly(ethylene glycol) ester prodrugs-design and in vivo effectiveness. $J$ Med Chem. 1996;39(2):424-431.

41. Venturoli D, Rippe B. Ficoll and dextran vs globular proteins as probes for testing glomerular permselectivity: effects of molecular size, shape, charge, and deformability. Am J Physiol Renal Physiol. 2005;288(4):F605-F613.

42. Harrington KJ, Rowlinson-Busza G, Syrigos KN, et al. Pegylated liposome encapsulated doxorubicin and cisplatin enhance the effect of radiotherapy in a tumor xenograft model. Clin Cancer Res. 2000;6(12):4939-4949.

43. Harrington KJ, Mohammadtaghi S, Uster PS, et al. Effective targeting of solid tumors in patients with locally advanced cancers by radiolabeled pegylated liposomes. Clin Cancer Res. 2001;7(2):243-254.

44. Liu Z, Liu LL, Yang TT. Comparative study of myocardial infarction models in two kinds of rat. Prog in Vet Med. 2010;31(4):19-25.
International Journal of Nanomedicine

\section{Publish your work in this journal}

The International Journal of Nanomedicine is an international, peerreviewed journal focusing on the application of nanotechnology in diagnostics, therapeutics, and drug delivery systems throughout the biomedical field. This journal is indexed on PubMed Central, MedLine, CAS, SciSearch ${ }^{\circledR}$, Current Contents ${ }^{\circledR} /$ Clinical Medicine,

\section{Dovepress}

Journal Citation Reports/Science Edition, EMBase, Scopus and the Elsevier Bibliographic databases. The manuscript management system is completely online and includes a very quick and fair peer-review system, which is all easy to use. Visit http://www.dovepress.com/ testimonials.php to read real quotes from published authors. 